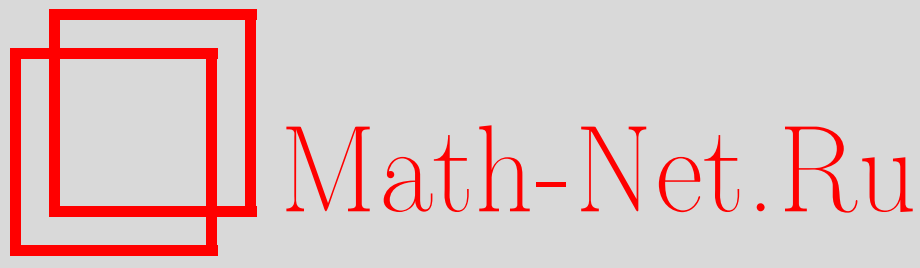

Общероссийский математический портал

С. Ю. Оревков, Проективные коники и $M$-квинтики в общем положении с максимально пересекающейся парой овалов, Матем. заметки, 1999, том 65, выпуск 4, 632-635

DOI: https://doi.org/10.4213/mzm1093

Использование Общероссийского математического портала Math-Net.Ru подразумевает, что вы прочитали и согласны с пользовательским соглашением http://www. mathnet.ru/rus/agreement 
Параметры загрузки:

IP: 18.208 .226 .222

26 апреля 2023 г., 15:06:51

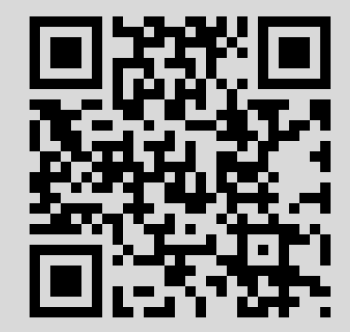




\section{ПРОЕКТИВНЫЕ КОНИКИ И $M$-КВИНТИКИ В ОБЩЕМ ПОЛОЖЕНИИ С МАКСИМАЛЬНО ПЕРЕСЕКАЮЩЕЙСЯ ПАРОЙ ОВАЛОВ}

\section{С. Ю. Оревков}

В настоящей работе продолжается исследование плоских вещественных приводимых кривых степени 7, начатое в [1]-[3]; дается изотопическая классификация кривых, указанных в заглавии. Мы используем те же методы, что и в [4], [3]. При этом, как оказалось, случай коники и квинтики более "регулярен", чем случай кубики и квартики: все запреты удается доказать по одной схеме, то же относится и к построениям. Мы придерживаемся тех же обозначений, что и в [3], и часто будем опускать подробности, которые можно найти в [3].

1. Формулировка результата. Далее $C_{n}$ есть множество вещественных точек $M$-кривой степени $n$ на $\mathbb{R P}^{2}$. Напомним, что $M$-квинтика $C_{5}$ состоит из семи гладких окружностей. Среди них шесть овалов (изотопных конике), расположенных вне друг друга, и одна нечетная ветвь $J_{5}$ (изотопная прямой). Всюду в данной работе мы предполагаем, что один из овалов $M$-квинтики $C_{5}$ (обозначим его через $O_{5}$ ) имеет десять различных точек пересечения с коникой $C_{2}$. Тогда по теореме Безу все пересечения должны быть трансверсальньми, а остальные пять овалов не могут пересекаться с $C_{2}$, эти овалы мы будем назьвать свободными.

Выбирая различными способами две точки в разных компонентах $\mathbb{R P}^{2} \backslash\left(C_{2} \cup C_{5}\right)$ и учитьвая, что проходящая через эти точки прямая не может пересекать $C_{n}$ более чем в $n$ точках, несложно проверить (см. [1], [2]), что с точностью до изотопии $C_{5}$ должна быть расположена относительно $C_{2}$ одним из десяти способов, изображенных на рис. 1 , где нечетная ветвь $J_{5}$ не нарисована и $\alpha, \beta$ - априори произвольные неотрицательные целые числа такие, что $\alpha+\beta=5$ (это количества свободных овалов в соответствующих областях).

Работа выполнена при частичной поддержке Российского фонда фундаментальных исследований, грант № 98-01-00794. 


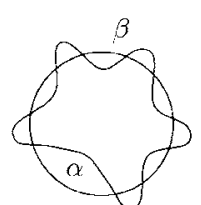

$\alpha=1,2,3$

$<+++$ +

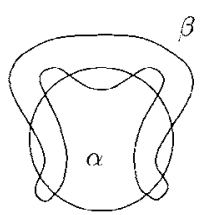

$\alpha=0,1,2,3$

$<+--+>$

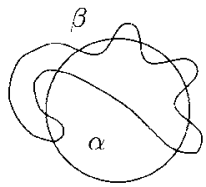

$\alpha=1,2,3,4$

$<-++$ +

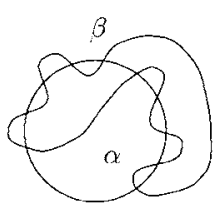

$\alpha=1,2,3,4$

$<++-->$

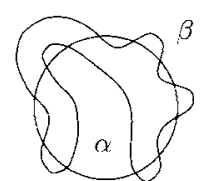

$\alpha=0,1,2,3,4$

$<+-t+>$

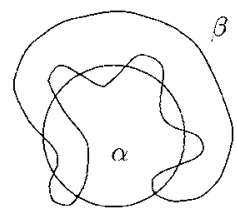

$\alpha=1,2,3,4$

$<+--->$

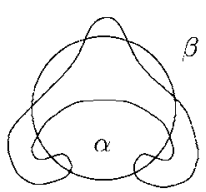

$\alpha=2,3,4,5$

$<-++->$

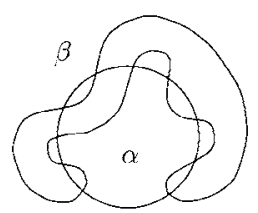

$\alpha=1,2,3,4,5$

$<-+-->$

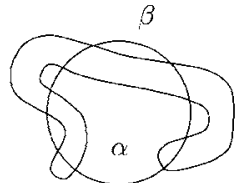

$\alpha=0,1,2,3,4,5$

$<+-+->$

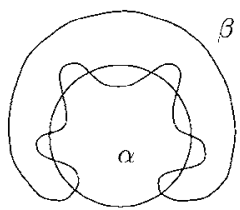

$\alpha=2,3,4$

$<--->$

Рис. 1

Цель настоящей статьи - доказать, что реализуемы те и только те расположения, которые отвечают значениям $\alpha$, перечисленным на рис. 1.

2. Построения. По аналогии с [3] расположения $C_{5}$ относительно $C_{2}$ (удовлетворяюшие условиям, перечисленным в п. 1) мы будем кодировать следующим образом. Словом $w=\left\langle s_{1} \ldots s_{n}\right\rangle$, $s_{k} \in\{+,-\}$, будем кодировать расположение $O_{5}$ относительно $C_{2}$, составленное из блоков $B\left(s_{1}\right), \ldots, B\left(s_{n}\right)$ в соответствии с рис. $2 \mathrm{a} ;$ блоки $B(+)$ и $B(-)$ изображены на рис. 2 . Параметр $\alpha$ будем указьвать в фигурных скобках после слова $w$.

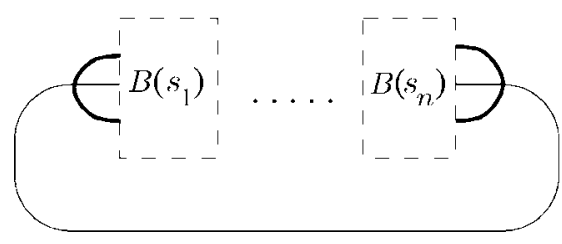

a

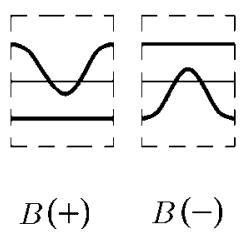

6

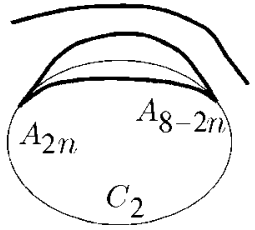

B

Рис. 2

Все реализуемые расположения строятся сглаживанием подходящих особых кривых. Обозначим через $2 n\{\alpha\}, n=0,1,2, \alpha=0,1$, расположение особой трехкомпонентной квинтики относительно коники $C_{2}$, изображенное на рис. 2в: квинтика имеет две особые точки типов $A_{2 n}, A_{8-2 n}$ (по соглашению $A_{0}$ - гладкая точка), лежащие на одной четной ветви, и свободный овал квинтики расположен внутри $C_{2}$ при $\alpha=1$ и снаружи при $\alpha=0$.

ЛЕмма. Все шесть расположсений $2 n\{\alpha\}, n=0,1,2, \alpha=0,1$, реализуемы алгебраическими кривыми.

ДокАЗАТЕльство. Пусть кривая $C$ в точке $p$ неособа и не имеет перегиба. Обозначим через $f_{C, p}$ квадратичное бирациональное преобразование $(u, v) \mapsto\left(u, v-u^{2}\right)$, где $(u, v)$ - аффинная карта, в которой бесконечно удаленная прямая касается $C$ в точке $p$, причем $C$ имеет возле $p$ разложение $v=u^{2}+O(u), u \rightarrow \infty$. 


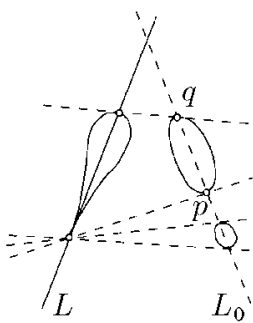

a

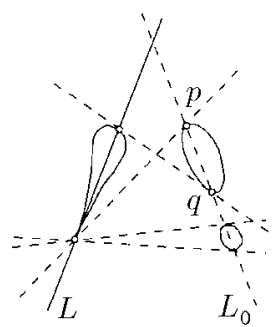

6

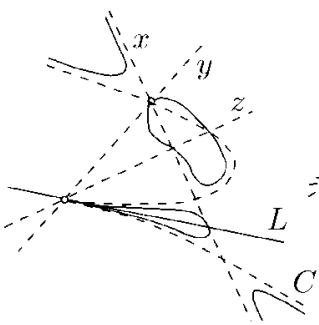

B

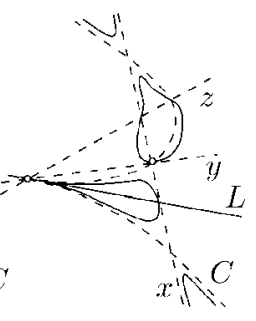

$\Gamma$

Рис. 3

$0\{\alpha\}$ и $2\{\alpha\}$. Пусть $Q:=L^{2} L_{0}^{2}+\varepsilon l_{1} l_{2} l_{3} l_{4},|\varepsilon| \ll 1,-$ каспидальная трехкомпонентная квартика и $p, q$-ее точки касания с двумя из прямых $l_{i}$ (см. рис. 3 а, 3б). Тогда $f_{Q, p}(Q) \cup f_{Q, p}(L)$ реализует расположение $0\{1\}$ в случае рис. 3 а и $0\{0\}$ в случае рис. $3 б$, а $f_{Q, q}(Q) \cup f_{Q, q}(L)$ реализует расположение $2\{0\}$ в случае рис. За и $2\{1\}$ в случае рис. $3 б$.

$4\{\alpha\}$. Пусть $C$ - каспидальная кубика, расположенная относительно координатных осей, как показано на рис. 3 в, $3 г$, и $Q:=x C+\varepsilon y z L^{2}-$ каспидальная квартика. Пусть hу - гиперболизм, т.е. бирациональное преобразование $\mathrm{hy}(x: y: z)=\left(x^{2}: x y: y z\right)$. Тогда $\mathrm{hy}(Q) \cup \mathrm{hy}(L)$ реализует расположение $4\{1\}$ в случае рис. 3 в и $4\{0\}$ в случае рис. 3 г.

В [3] показано, что для любого выбора знаков $s_{1}, \ldots, s_{n}, s_{1}^{\prime}, \ldots, s_{4-n}^{\prime}$ существует возмущение кривой $2 n\{\alpha\}$ вида $\left\langle s_{1} \ldots s_{n} s_{4-n}^{\prime} \ldots s_{1}^{\prime}\right\rangle\left\{\alpha+\alpha_{0}\right\}$, где $\alpha_{0}$ - число плюсов в последовательности $-s_{1}, s_{2}, \ldots,(-1)^{n} s_{n},(-1)^{n} s_{4-n}^{\prime}, \ldots, s_{2}^{\prime},-s_{1}^{\prime}$. Несложно проверить, что это дает все расположения на рис. 1 .

ЗАмеч АниЕ. Четыре расположения $\langle++++\rangle\{\alpha\}\langle----\rangle\{\alpha+1\}, \alpha=2,3$, можно построить элементарно: надо в качестве $C_{5}$ взять подходящее возмущение двух коник (одна из них будет $C_{2}$ ) и прямой.

3. Запреты. Не реализованные выше значения $\alpha$ для случая $\langle++++\rangle$ запрещены методом комплексных ориентаций $[1, \mathrm{VII}]$ (см. также [3, 3.1]). Во всех других случаях найдется точка $p$, лежащая в области $\alpha$ в выпуклой оболочке овала $O_{5}$ и, следовательно, вне свободных овалов квинтики (ср. с проекцией III в $[3,3.2 .4])$. Выберем афффинные координаты $(x, y)$, в которых $p$ - бесконечно удаленная точка прямых $x=$ const и бесконечно удаленная прямая в пяти точках пересекает $O_{5}$. Тогда $C_{2}, O_{5}$ и $J_{5}$ расположены относительно этих координат, как показано на рис. 4 , где блоки $B\left(s_{i}\right)$ те же, что и на рис. 2 б.
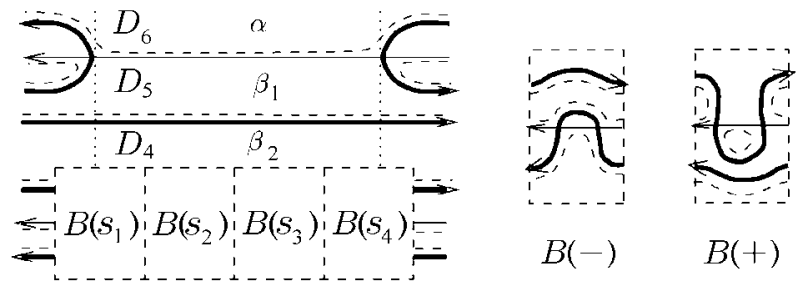

$$
B(-) \quad B(+)
$$

Рис. 4

Поскольку вертикальная прямая не может пересекать $C_{5}$ более чем в пяти точках, свободные овалы квинтики должны лежать в полосе между вертикальными касательными к $O_{5}$ (обозначим 
ее через $D$ ). Пусть $D_{4}, D_{5}, D_{6}$ - три верхние компоненты связности множества $D \backslash\left(C_{2} \cup O_{5}\right)$ (см. рис. 4). Тогда $\alpha$ овалов лежат в $D_{6}$ и $\beta$ овалов - в $D_{5} \cup D_{4}$. Занумеруем свободные овалы квинтики слева направо, и пусть $D_{i_{j}}$ - полоса, в которой лежит $j$-й овал, $j=1, \ldots, 5$. Положим $\delta \alpha=\sum_{i_{j}=6}(-1)^{j}, \delta \beta_{k}=\sum_{i_{j}=6-k}(-1)^{j}, k=1,2$.

Из формулы комплексных ориентаций для $C_{5}$ (см. [5]) и теоремы Фидлера о чередовании ориентаций [6] следует, что

$$
\delta \alpha+\delta \beta_{1}-\delta \beta_{2}=0
$$

Выберем комплексные ориентации на $C_{2}$ и $C_{5}$ так, чтобы $C_{2}$ и $O_{5}$ были ориентированы противоположно относительно $J_{5}$. Пусть $F$ - кривая степени 7 , получающаяся из $C_{2} \cup C_{5}$ сглаживанием двойньх точек в соответствии с выбранными ориентациями (изображена пунктиром на рис. 4). Тогда число овалов кривой $F$ равно $2 P+7$, где $P$ - число плюсов в слове $\left\langle s_{1} s_{2} s_{3} s_{4}\right\rangle$, кодирующем рассматриваемое расположение $C_{2} \cup O_{5}$. Поэтому формула комплексных ориентаций для $F$ дает

$$
\varepsilon\left((1+2 \varepsilon) \delta \alpha+\delta \beta_{1}-\delta \beta_{2}\right)=2 P-5,
$$

где $\varepsilon= \pm 1-$ знак овала $O_{5}$ относительно нечетной ветви $J_{5}$. Добавляя к $(1),(2)$ очевидное соотношение $\delta \alpha+\delta \beta_{1}+\delta \beta_{2}=-1$ и решая получившуюся систему уравнений, находим

$$
\delta \alpha=q-2, \quad \delta \beta_{1}=1-q, \quad \delta \beta_{2}=0, \quad \text { где } q=P+\frac{\varepsilon-1}{2} .
$$

Для расположений $\left\langle s_{1} s_{2} s_{3} s_{4}\right\rangle\{\alpha\}$, не реализованных в п. 2, все наборы чисел $i_{1}, \ldots, i_{5}$, для которых сушествует $\varepsilon= \pm 1$, при котором выполнены соотношения (3), таковы (последней колонке этой таблицы отвечают расположения, запрещенные в $[1, \mathrm{VII}]$ методом комплексных ориентаций; в

\begin{tabular}{|c|c|c|c|c|c|}
\hline $\begin{array}{l}\langle-+++\rangle\{0\} \\
\langle--++\rangle\{0\}\end{array}$ & $\langle-++-\rangle\{1\}$ & $\langle-++-\rangle\{0\}$ & $\langle+--+\rangle\{4\}$ & $\begin{array}{l}\langle++--\rangle\{5\} \\
\langle+--+\rangle\{5\} \\
\langle+---\rangle\{5\}\end{array}$ & $\begin{array}{l}\langle++++\rangle\{0\} \\
\langle++++\rangle\{4\} \\
\langle++++\rangle\{5\}\end{array}$ \\
\hline 44445 & 44446 & & & & $\langle+++-\rangle\{5\}$ \\
\hline 44544 & 44556 & & & & $\langle++-+\rangle\{5\}$ \\
\hline 44555 & 44644 & & & & $\langle+---\rangle\{0\}$ \\
\hline 45545 & 44655 & & & & $\langle-+--\rangle\{0\}$ \\
\hline 54444 & 45546 & 44445 & & & $\langle----\rangle\{0\}$ \\
\hline 54455 & 45645 & 44544 & & & $\langle----\rangle\{1\}$ \\
\hline 54554 & 54456 & 44555 & & & $\langle----\rangle\{5\}$ \\
\hline 55445 & 55446 & 45545 & & & \\
\hline 55544 & 55556 & 54455 & 56666 & & нет \\
\hline 55555 & 55655 & 55555 & 66566 & 66666 & решений \\
\hline
\end{tabular}
частности, мы видим, что в [1] на рис. 16 была опечатка: вместо $\alpha \neq 2$ для пятой модели должно быть $\alpha \neq 5$ для второй модели):

(в столбцах 2-4 учтена симметричность слов $\langle-++-\rangle,\langle+--+\rangle)$. Проверяя для каждого из перечисленных 41 случаев неравенство Мурасуги-Тристрама так, как это было сделано в [3], убеждаемся, что все они нереализуемы.

Автор благодарит Г. М. Полотовского за полезные обсуждения. Автор выражает признательность Математическому институту им. Макса Планка за гостеприимство. 


\section{СПИСОК ЦИТИРОВАННОЙ ЛИТЕРАТУРЫ}

1. Polotovskii G. M. // Lecture Notes in Math. 1992. V. 1524. P. 52-74. 2. Polotovskii G.M. On the classification of decomposable 7th degree curves // Contemp. Math. 1999. (to appear). 3. Оревков С. Ю., Полотовский Г. М. Проективные $M$-кубики и $M$-квартики в общем положении с максимально пересекающейся парой овалов // Алгебра и анализ. (в печати). 4. Orevkov S. Yu. Link theory and oval arrangements of real algebraic curves // Topology. (to appear). 5. Рохлин В. А. // УМН. 1978. Т. 33. № 5. С. 77-89. 6. Фидлер Т. // Изв. АН СССР. Сер. матем. 1982. Т. 46. С. 853-863.

Математический институт им. В. А. Стеклова РАН

Поступило

E-mail : orevkov@mi.ras.ru

02.11 .98 